\title{
Experimental attempts to extend the current preimplantation genetic diagnosis with individual karyotypization of human blastomeres
}

\author{
Daniel HLINKA $^{\mathrm{a}}$, Marek DudÁš ${ }^{\mathrm{b} *}$, Miroslav HERMAN $^{\mathrm{a}}$, Ivan KALINA ${ }^{\mathrm{b}}$ \\ a Assisted Reproduction Center, L. Pasteur University Hospital, Košice, Slovakia \\ ${ }^{\mathrm{b}}$ Department of Medical Biology, P.J. Šafárik University, Trieda SNP 1, \\ 04066 Košice, Slovak Republic
}

(Received 5 September 2000; accepted 29 December 2000)

\begin{abstract}
The drug-induced chromosome condensation using okadaic acid, a potent protein phosphatase inhibitor, was studied in day 1 to day 4 (D1-D4) spare human preimplantation embryos. In order to obtain cells for genetic tests, we developed a modified displacement blastomere biopsy method. During the okadaic acid treatment, approximately $40 \%$ of biopsied cells were lost due to heavy changes of the plasma membrane; this detrimental effect of okadaic acid differed markedly with the respect to the age of embryos. In comparison with the natural embryonic mitotic index, day 1 and day 2 embryonic cells gave increased yields of chromosome spreads (up to $51 \%$ of the initial D1-D2 cell number); on days 3 and 4 we were not able to obtain from surviving cells more than $31 \%$ blastomeres with condensed chromosomes (9\% of total D3-D4 cell number). All chromosome spreads were successfully used for recycling in G-banding and subsequent FISH analysis.

assisted reproduction / blastomere biopsy / chromosome aberration / fluorescent in situ hybridization (FISH) / G-banding / human early embryo / karyotype / okadaic acid / preimplantation genetic diagnosis

Résumé - Effort expérimental orienté vers l'élargissement du diagnostic génétique de préimplantation de la caryotypisation de différents blastomères humains. Nous avons étudié la condensation des chromosomes de différentes cellules qui étaient isolées des embryons humains aberrants de préimplantation agées de 1-4 jours (D1-D4), provoquée par l'acide okadaic - un inhibiteur fort des phosphatases de protéines. Nous avons utilisé la méthode modifiée de la biopsie d'un blastomère. Cca $40 \%$ de cellules a disparu à cause de grandes modifications de la membrane cytoplasmique au cours de l'action de l'acide okadaic. Chez les cellules des embryons de 1 et de 2 jours, nous avons obtenu le nombre élevé de chromosomes (jusqu'à $51 \%$ du nombre initial de cellules). En ce qui concerne les cellules de 3 et 4 jours, nous n'avons réussi à obtenir que $31 \%$ de blastomères avec
\end{abstract}

* Correspondence and reprints

E-mail: dudasm @ central.medic.upjs.sk 
les chromosomes condensés ( $9 \%$ du nombre total de cellules concentrées). Tous les échantillons de chromosomes ont bien recyclé dans les procédures de «G-banding » avec l'analyse FISH successive.

aberration du chromosome / acide okadaic / biopsie d'un blastomère / caryotypisation / diagnostic génétique de préimplantation / embryon humain / G-bande / hybridisation fluorescente in situ (FISH) / reproduction assistée

\section{INTRODUCTION}

Preimplantation genetic diagnosis (PGD) represents the most recent progress in human genetics and reproductive medicine. It can be performed only in conjunction with assisted reproduction technologies (AR, ART), because only the in vitro period (starting from the isolation of gametes and ending with the embryotransfer after several days of the early embryo development) gives the possibility to separate polar bodies (PB; PB1 and PB2) from oocytes and zygotes, or one to two cells from developing preimplantation embryos, for the use in genetic tests.

Presently, the day 3 (D3) of human embryo development is considered to be the most convenient time for the embryo biopsy, because the number of cells reaches 6 to 10 , and also because this is the last time when blastomeres are not mutually connected (precompaction developmental stage). It is of a great importance to finish genetic tests and embryo selection within 24 (maximally 72) hours after blastomere removal (i) to meet the receptive period of the mother's uterus, and (ii) to meet the embryological laboratory's culture protocols - successful blastocyst (D5-D7) cultivation is not yet optimal. Moreover, on D5-D7 the zona pellucida $(\mathrm{ZP})$ is thickened and broken while embryo hatching occurs, after which the further cultivation is impossible because of the need of embryo implantation into the endometrium. Furthermore, (iii) it may be more acceptable and convenient to prefer genetically unaffected embryos for prolonged culture and/or cryopreservation, in dependence on the particular country's legal or ethical background.
Three main micromanipulation methods are currently employed in embryo biopsy: (i) the classical "zona drilling", i.e. making an opening in ZP with a stream of strongly acidic Tyrode's solution followed with a blastomere aspiration using a polished pipette; the negatives of this method are a big defect in the ZP and the subzonal space acidification, potentially harmful to embryo; (ii) ZP penetration and blastomere aspiration using the same beveled pipette; the pipette's diameter must be carefully calibrated and the pipette's tip must be sharp enough to cut the ZP, but sharp pipettes often cause a blastomere damage; (iii) partial ZP dissection (mechanical or lasermade) and subsequent blastomere aspiration with a polished pipette; laser zona dissectors are very strongly commercially propagated, but expensive, and the negative influence of carbonization by-products is unknown; using a mechanical dissection, the dissecting instrument must be replaced with a polished pipette for aspiration (time consuming). In the methodical part of this article we describe a very simple, modified microbiopsy method raised from experiences with the intracytoplasmic sperm injection (ICSI) [14].

Current clinical PGD is based on DNA analysis, i.e. PCR or F-PCR (polymerase chain reaction and fluorescent PCR), and on molecular cytogenetic methods like FISH and PRINS (fluorescence in situ hybridization, primed in situ synthesis). Experimental approaches comprise mRNA and mtDNA analysis, $\mathrm{CGH}$ (comparative genomic hybridization), protein assays, and conventional cytogenetics, sometimes. 
PCR-based methods are primarily suitable for targeted gene analyses, but also some data touching chromosome number may be obtained in some rare cases (sex chromosome composition, aneuploidy detection based on individual DNA polymorphisms and/or quantitative PCR). Top PGD laboratories have a relatively low amplification failure, and sufficiently high diagnostic sensitivity and reliability. After preparation of nuclei for the DNA analysis, no chromosome structural information (translocations, inversions, duplications, a majority of aneuploidies etc.) is available. In general, no more than a few loci can be simultaneously analyzed in a single PCR reaction with one nucleus [10].

Hybridization-based cytogenetic methods are suitable for a rapid detection of chromosome number. Clinical FISH aneuploidy analysis is limited to approximately 10 chromosome pairs in one procedure due to a lack of various fluorescent dyes. The use of pancentromeric probes falls to give a total chromosome number because of frequent signal overlapping in small nuclei. The most important limitation of cytogenetic approaches is the minimal probability of performing the blastomere biopsy just during the metaphase stage of the cell cycle. Usually, fixed nuclei are of the interphase morphology, and structural or numerical chromosome analysis is impossible. Only in several specific cases of known predisposition, some chromosome rearrangements were detected in an interphase chromatin using custom-constructed breakpoint-spanning probe [3]. In general, blastomere nuclei are not suitable for a total chromosome enumeration neither for the karyotype evaluation; metaphase spreads are the rare occasional findings only.

Repeated FISH on a single fixed nucleus is technically possible [17], but this way requires repeating a long FISH procedure several times to count all chromosomes in an interphase chromatin; also the hybridization efficiency decreases in consecutive
FISH rounds. To increase the value of blastomere biopsy, there were some partly successful experimental protocols developed for recycling a single fixed nucleus in hybridization procedures and subsequently in PCR analysis [19] to join a fragment of cytogenetic findings with a fragment of mutation information obtainable from a single cell.

Conventional cytogenetic protocols based on the culture of biopsied blastomeres, their cleavage and colchicine treatment, and subsequent karyotype analysis are laborious and time consuming. Also they are not acceptable for the clinical PGD due to a frequent cell death of isolated blastomeres, and because of a general instability of the early embryos' immature mitotic apparatus, which can potentially lead to a misdiagnosis after individual blastomere cultivation. But condensed chromosomes can give us the most complete information on structural and numeric chromosome aberrations that are very frequent in human early embryos, and which are thought to be the most often causes of reproductive failure in humans.

Further research is needed (i) to introduce more reliable cytogenetic methods for a conventional or fluorescent karyotype analysis, (ii) to increase the whole genomic DNA amplification enough to prepare sufficient amounts of DNA for multiple gene analyses or gene chip technologies, and (iii) eventually to improve the cytogenetic-PCR recycling protocols.

Our preliminary work in the field of developing a karyotype analysis protocol for PGD is based on following facts:

(i) Starting from the first cell division of the zygote, naturally condensed chromosomes of early mammalian embryos, including human ones, are similar to mitotic chromosomes common in peripheral blood lymphocytes. They condense during mitosis, and in some cases they may be conventionally G-banded. These findings were collected from experiments, in which embryos of various developmental stages or their 
individual blastomeres were fixed randomly or in the time of expected $M$ phase of the cell cycle, with or without a long (18 to $20 \mathrm{~h})$ colchicine exposure [26, 27].

(ii) Interphase chromatin can undergo a rapid condensation in vivo under special biological circumstances. Many investigators obtained metaphase spreads in fusion experiments with mitotic cells or with oocytes containing high levels of the maturation-promoting factor (MPF) $[4,7,16,25]$

(iii) A decade ago, the maturation stimulating effect of okadaic acid on animal oocytes was described, and MPF was shown to play the key role in this process, which involves chromosome condensation [21, 22]. Several experiments based on chromosome condensation induced by okadaic acid treatment (OAT) were performed in mammalian oocytes and D1 embryos (zygotes) $[9,25]$. The most recent success is the karyotypization of polar bodies and blastomere nuclei, reached after their incorporation (using fusion or injection) into oocytes or zygotes, that was in some cases followed with a subsequent OAT [4].

(iv) Drug-induced chromosome condensation is the newly described phenomenon, observed after the treatment of interphase cells from adult mammals with various potent phosphatase inhibitors: okadaic acid, already mentioned above (OA, isolated from Prorocentrum concavum, inactivates Ser/Thr protein phosphatase (PP) types in the order: $2 \mathrm{~A}>1>2 \mathrm{~B}$ ), fostriecin (Streptomyces pulveraceus, specific for PP type 2A), or calyculin A (Discodermia calyx, $\mathrm{PP} 2 \mathrm{~A}=1 \gg 2 \mathrm{~B}$ ). Treated somatic cells condensed their chromosomes in almost all cell cycle phases, and very high (from $16 \%$ up to $99 \%$ ) ratios of nuclei with condensed chromosomes were reported in some somatic and tumor cell lines $[5,8]$. The major advantages of this approach were: rapid obtaining of chromosome spreads from tissues and cells that have given condensed chromosomes never before, and immediate obtaining the chromosome spreads in unusual cell cycle stages. The efficiency of this approach and the quality of chromosome spread varied markedly in different cell types. To our knowledge, the efficiency of this method has not yet been studied in embryonic cells.

Inspired by these very promising experiments, we decided to study the druginduced chromosome condensation efficiency using okadaic acid treatment (OAT) applied on free, non-fused human early embryo blastomeres, in correlation to their developmental age (D1 and D2, D3 and D4). We also present our experience on how to fix and manage a single chromosome spread on a microscope slide during diagnostic procedures. A detailed description of repeated use of the same single-cell chromosome spread for G-banding and subsequent FISH analysis in the field of preclinical PGD is given here.

\section{MATERIALS AND METHODS}

\subsection{Embryos}

D1 to D4 live spare human embryos, i.e. those showing haploidy, polyploidy, morphological abnormalities (uneven divisions, fragmentation, multinuclearity), and/or growth retardation (assessed as a low cell number in correlation to the day of development) or cleavage arrest, were used for this study. All embryos were obtained from our clinical assisted reproduction program after being legally excluded from further cultivation and embryotransfer in accordance with legal, ethical and clinical criterions. Embryos were concepted by the means of classical IVF (in vitro fertilization) or ICSI, and were not selected with the respect of their origin.

\subsection{Blastomere biopsy}

In some cases, whole embryos were disintegrated to individual cells after an enzymatic $\mathrm{ZP}$ removal with $2 \%$ pronase $\mathrm{E}$ 
(EC 3.4.24.31 from Streptomyces griseus, Sigma) in $0.9 \% \mathrm{NaCl}$ solution, $\mathrm{pH} 7.3$, or after a ZP hydrolysis with the use of acidic Tyrode's solution $(0.08 \mathrm{~g} \mathrm{NaCl}, 0.002 \mathrm{~g}$ $\mathrm{KCl}, 0.0026 \mathrm{~g} \mathrm{CaCl}{ }_{2} \cdot 2 \mathrm{H}_{2} \mathrm{O}, 0.01 \mathrm{~g}$ $\mathrm{MgCl}_{2} \cdot 6 \mathrm{H}_{2} \mathrm{O}, 0.01 \mathrm{~g} \mathrm{D}$-glucose, $0.04 \mathrm{~g}$ polyvinylpyrrolidone, deionized water to $10.0 \mathrm{~mL}, \mathrm{pH}$ adjustment with $\mathrm{HCl}$ to 2.1-2.5). After the use of the zona digestion, embryos were washed two times in a culture medium. The other embryos were microbiopsied using the modified singleneedle displacement method consisting of $\mathrm{ZP}$ dissection followed by blastomere isolation by the means of a hatching-like expulsion (see below). When the expulsion of the last cells of spare embryos from ZP was difficult, remaining blastomeres were isolated after the ZP digestion, like in previous cases.

Blastomere biopsy (Fig. 1) was performed with the use of a hydraulic micromanipulator (Leitz, Germany). Cleaving embryos were transferred into a small drop $(10 \mu \mathrm{L})$ of a culture medium covered with preequilibrated paraffin oil. All manipulations were carried out on a heated microscope stage. Embryos were immobilized on a fire-polished holding pipette by a gentle suction. Self-made glass microneedle was tangentially pricked into the ZP, slightly entering the very peripheral subzonal space and passing out the ZP not far from the entry point. Liberated from the holder pipette, at this moment the embryo is fixed to the glass needle piercing its ZP. A gentle pressure of the needle against the holder causes a crack of the ZP region situated between the two instruments - the moment of the partial zona dissection (PZD). The diameter of the hole made in $\mathrm{ZP}$ should be approximately two thirds of the diameter of the cell to be removed. The holder has immobilized embryo once more, now $90^{\circ}$ from the dissection site. The same pipette that was used for opening the zona was filled with a medium (dissecting pipette was slightly opened by breaking its tip against the holder to allow medium aspiration), and then inserted under the zona at the pole opposite to the dissected crack. Subzonal injection of a sterile culture medium increases the intrazonal pressure, the situation resulting in the herniation of the blastomere that is mostly adjacent to the dissected opening. Intact cell or desired number of cells may be expulsed this way (except for some last residual cells - but this fact is irrelevant in the context of clinical PGD, when only one or two cells are needed).

\subsection{Okadaic acid treatment}

Individual blastomeres were cultured in medium supplemented with okadaic acid to the final concentrations $0.25,0.5,1,2,4,6$, or $10 \mu \mathrm{mol} \cdot \mathrm{L}^{-1}$. (Stock solution $2.5 \mathrm{mmol} \cdot \mathrm{L}^{-1}$ in absolute DMSO, stored at $-15^{\circ} \mathrm{C}$, was diluted with the culture medium to final concentrations; the final concentration of DMSO was maximally $0.1 \%$ for $0.25 \mathrm{mM}$ $\mathrm{OA}$, and maximally $4 \%$ o for $10 \mu \mathrm{M} \mathrm{OA}$ solutions. The rank of OA concentrations used in this work was designated in our preliminary experiments - data not shown.)

Ten microliters drops of drug containing media (supplemented with $15 \%$ of thermally inactivated maternal serum) were covered with preequilibrated paraffin oil and cultured with isolated blastomeres for 1 to $4 \mathrm{~h}$ at $37.0{ }^{\circ} \mathrm{C}$ in the atmosphere containing $5 \% \mathrm{CO}_{2}$. Cells were firstly placed in $2 \mu \mathrm{M} \mathrm{OA}$ medium. When changes of the plasma membrane occurred (seen as multiple vesiclelike exvaginations covering the whole surface of blastomeres), the cells were individually washed and transferred to a stronger or less concentrated OA medium, or to a normal cultivation medium, in accordance with the plasma membrane changes. The presence of the nuclear envelope (NE) and the state of nucleoli were noticed during the process every $30 \mathrm{~min}$ using the inverted microscope equipped with phase contrast optics. After NE and nucleoli disappeared, the cells were fixed. In later experiments, cytochalasin B [18] was added to the OA medium to a final concentration $5 \mu \mathrm{g} \cdot \mathrm{mL}^{-1}$ 


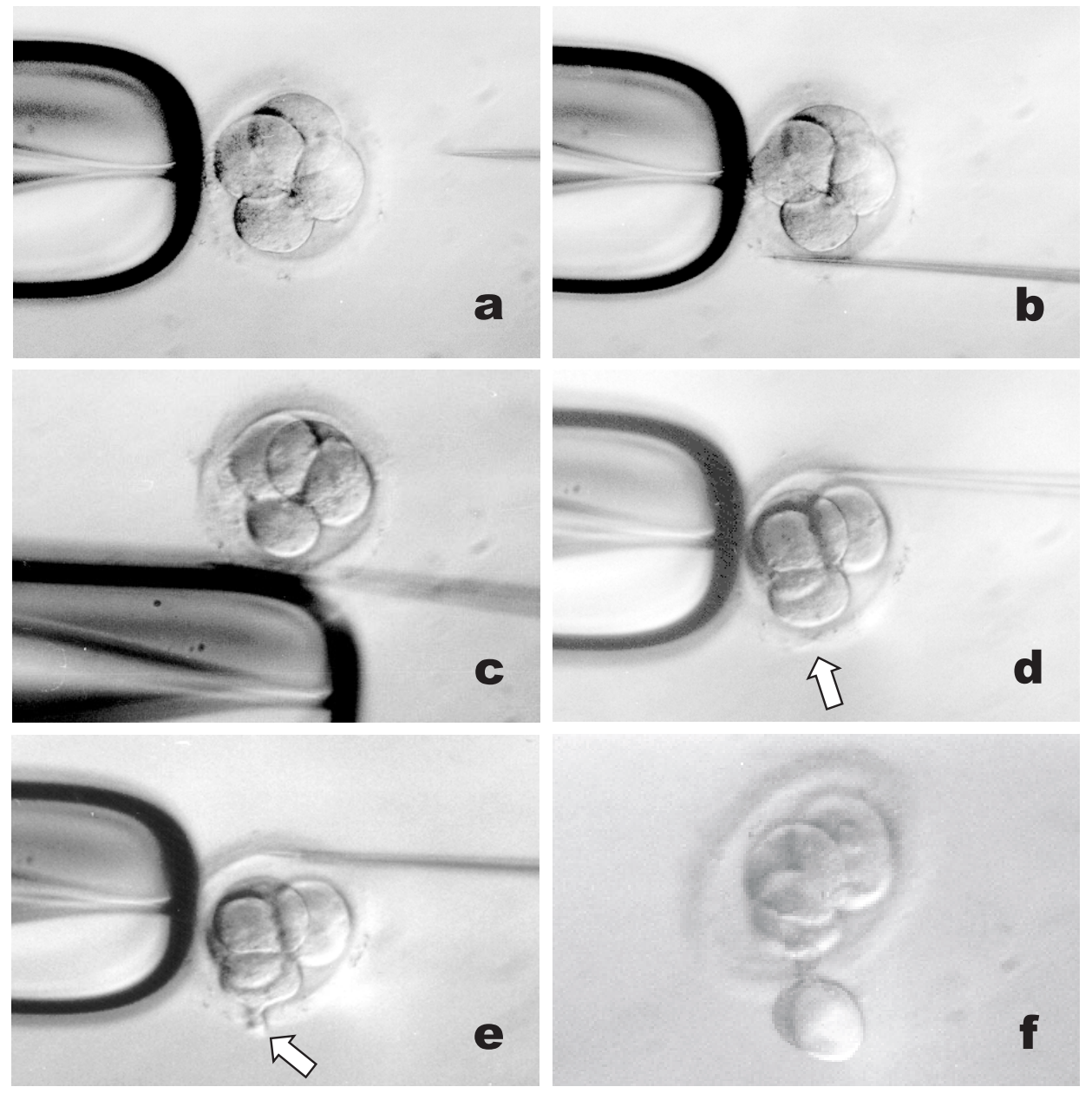

Figure 1. Individual steps of modified blastomere biopsy. This new method is easy to perform without the need of laser, acidic solutions, and pipette changing. (a) Human embryo is immobilized by a gentle suction of the holder pipette (left). On the right side there is the working pipette (dissecting glass needle). (b) Working pipette tangentially pierces the zona pellucida. (c) The moment of a partial zona dissection. Gentle pressure against the holder causes cutting the part of the zona situated between the needle and the holder. (d) Working pipette penetrates the zona again on the opposite pole of the embryo. The arrow indicates the dissected slit. (e) While injecting a sterile medium into the subzonal space, one blastomere herniates from the intrazonal space via the dissected opening (arrow). (f) Another embryo showing initial compaction. After the biopsy procedure, spontaneous constriction of the dissected site leads to a successful blastomere displacement either in initially compacting embryos. Separated blastomeres are used for genetic tests. 
to try to prevent severe membrane changes (see Sect. 4).

\subsection{Chromatin fixation}

After the OA treatment, blastomeres of disintegrated embryos or microbipsied blastomeres were hypotonized in $0.068 \mathrm{M} \mathrm{KCl}$ water solution for 0.5 to $10 \mathrm{~min}$, and fixed onto slides according the modified protocol based on the method after Tarkowski $[24,25]$. In brief, after hypotonic treatment, a short bath of cells in 1:1 methanol-acetic acid fixation mixture was followed with a suction of the cells into a pipette. The cell with a small amount of fixative was immediately dropped onto carefully cleaned microscope slides under stereomicroscopic control. Dropping sites were pre-marked on the opposite, lower side of slides. Some additional drops of fixative can be added when insufficient cytoplasm dissolution occurs. Slides were left to air-dry.

\subsection{Chromosome spread localization}

In the molecular genetic laboratory, slides were carefully observed using pseudo phase contrast with lowered condenser. All fixed objects were found in roughly pre-marked dropping sites and their exact position was marked with additional short color lines (Figs. 3a and 3b). 300-800 × 300-800 $\mu \mathrm{m}$ fine fields were manually delineated with a diamond needle, closely around all chromatin objects (Figs. 3c and 3d). Color marks were washed out in $70 \%$ ethanol and preparations were re-fixed and dehydrated in ascendant 70, 80, 90 and 99\% ethanol array, 2 min each at room temperature.

\subsection{G-banding}

Slides were immersed for $1.5 \mathrm{~min}$ at $60{ }^{\circ} \mathrm{C}$ in $2 \times \mathrm{SSC}$, pH $7.2(1 \times \mathrm{SSC}$ is standard saline citrate, $0.15 \mathrm{M} \mathrm{NaCl}$ with $0.015 \mathrm{M} \mathrm{Na}_{3}$-citrate in re-distilled water).
Slides then were rinsed with distilled water, air-dried and stained for 30-120 s with Wright's stain (Wright's stain - stock solution: $0.5 \mathrm{~g}$ Wright's powder mixed continuously $7 \mathrm{~h}$ with $200 \mathrm{~mL}$ of methanol, filtered, incubated for 7 days at $37^{\circ} \mathrm{C}$, then stored in dark at $4{ }^{\circ} \mathrm{C}$; phosphate buffer: $5.25 \mathrm{~g} \mathrm{Na}_{2} \mathrm{HPO}_{4} \cdot 2 \mathrm{H}_{2} \mathrm{O}$ and $4.13 \mathrm{~g} \mathrm{KH}_{2} \mathrm{PO}_{4}$, re-distilled water to $1000 \mathrm{~mL}, \mathrm{pH}$ 6.8; staining solution: freshly mixed stock solution and phosphate buffer 1:3). Finally, slides were quickly washed in water and air-dried.

We used this technique to overcome the trypsin digestion needed for a conventional G-banding procedure. Using Wright's stain and no trypsin, inappropriately banded or too dark chromosome spreads may be decolorized in methanol and re-banded; pale chromosomes may be directly re-banded. Both steps may be performed many times, but hot SSC treatment should not be repeated [2]. Gentle handling is of a vital importance for maintaining the chromosomes intact for the use in following diagnostic procedures

During microscopy, we avoided any contact of chromosomes with immersion oil, because the subsequent FISH procedure requires water solutions. That is why we modified the usual G-band evaluation: we mounted the banded preparations in glycerol drops under coverslips. Photographs were taken immediately (Figs. 3e and 3f), because prolonged exposure of banded chromosomes to glycerol caused a continuous decolorization (but no real damage to chromosomes). Then, slides were demounted, quickly washed in water, re-banded or dehydrated in an ascendant ethanol array for FISH.

\subsection{FISH procedure}

Slides were dehydrated in an ascendant alcohol array at room temperature, removed from alcohol and quickly air-dried, then placed for 3 min into closed Coplin jar filled with a warm denaturing solution $\left(74{ }^{\circ} \mathrm{C}\right.$; 
final concentrations: $70 \mathrm{vol} \%$ deionized formamide, $2 \times \mathrm{SSC}, \mathrm{pH} 7.4$ ), heated in tempered water bath situated near a vapor exhauster. Then, slides were immediately submersed into ice-cold $70 \%$ ethanol for $2 \mathrm{~min}$; 2 min dehydration in each 80, 90 and $99 \%$ ethanol followed. Slides were quickly air-dried (less than $2 \mathrm{~min}$ ), $2 \mu \mathrm{L}$ of probe solution per $150 \mathrm{~mm}^{2}$ hybridization area was added avoiding air bubbles, and coverslips were sealed with a rubber cement to prevent probe evaporation. We used directly labeled fluorescent Vysis ${ }^{\circledR}$ CEP Spectrum Orange $\mathrm{T}^{\mathrm{TM}} /$ Spectrum Green ${ }^{\mathrm{TM}}$ dual mixed DNA probe designed for simultaneous visualization of $\alpha$-satellite repeats of X chromosome centromeric region (red signal) and Y chromosome heterochromatin block on distal q arm (green signal). Slides were placed into humidified chamber for at least $2 \mathrm{~h}$ at $42{ }^{\circ} \mathrm{C}$ for hybridization.

\subsection{Posthybridization washes and mounting}

Coverslips with rubber cement were gently removed paying attention not to smear hybridization areas. Excessive and crosshybridized probe was removed using the rapid wash procedure: $20 \mathrm{~s}$ at $73{ }^{\circ} \mathrm{C}$ in $0.4 \times$ SSC, pH 7.2, then $20 \mathrm{~s}$ in $2 \times \mathrm{SSC}$ with $0.1 \%$ NP 40 detergent, $\mathrm{pH} 7.2$ at room temperature, and finally, $5 \mathrm{~s}$ slow agitation in re-distilled water to remove all free crystalloids. This final step is our own modification, which strongly prevents microcrystal appearance on a slide surface, and this way decreases the irregular mountant spreading under coverslips and minimizes the number of microscopic air-bubbles during the mounting step (see bellow). After air-drying the slides, the mountant Vysis ${ }^{\circledR}$ DAPI II ${ }^{\mathrm{TM}}$ with antifade properties (containing the counterstaining fluorescent dye 4',6-diamidino-2phenylindole dihydrochloride $n$-hydrate, DAPI) was dropped on coverslips, and these were mounted upon hybridization areas. The molecular complex DAPI-DNA gives a strong blue fluorescence in $\lambda=452-470 \mathrm{~nm}$ when excited with UV light of $\lambda=350-367 \mathrm{~nm}$. This dye stains any present DNA and helps to find single chromosomes on the slide as far as to see if any signal observed lies within DNA mass (usually valid signals) or not (probably dirt, which also usually slightly varies in color and shape). After the mountant has completely spread in the capillary space under the coverslip, excessive liquid was removed by gentle towel pressure. The coverslip edges were firmly sealed with a nail varnish to prevent any contact of the harmful mountant compounds with the skin.

\subsection{Signal detection}

Preparations were observed under a mercury burner light using the fluorescence microscope Olympus ${ }^{\circledR}$ BX60. Specific triple band epifluorescence was used with the aligned optical filter set for DAPI/FITC/ TRITC (Chroma Technology Corp.). Photographic documentation was done with the Olympus ${ }^{\circledR}$ photomicrography system PM20 using manual exposure corrections and ASA/ISO 800-1600 Kodak ${ }^{\circledR}$ Ektapress Gold II or Kodak ${ }^{\circledR}$ Gold Zoom color negative films. For the publication purposes, photographs were scanned, and the contrast was enhanced using Microsoft ${ }^{\circledR}$ Photo Editor.

\section{RESULTS}

Embryos were divided into 2 groups in accordance with their age (D1 and D2 group, D3 and D4 group).

Out of 35 cells (one- and two-cell stage embryos on D1 and D2), 29 (83\%) survived the OA treatment; 18 of 35 cells $(51 \%)$ underwent NEBD and chromosome condensation within $4 \mathrm{~h}$.

Out of 64 cells (more than two-cell stage embryos on D3 and D4), only 19 (29\%) survived the OA procedure. In 6 of 19 blastomeres $(31 \%)$, countable spreads were 

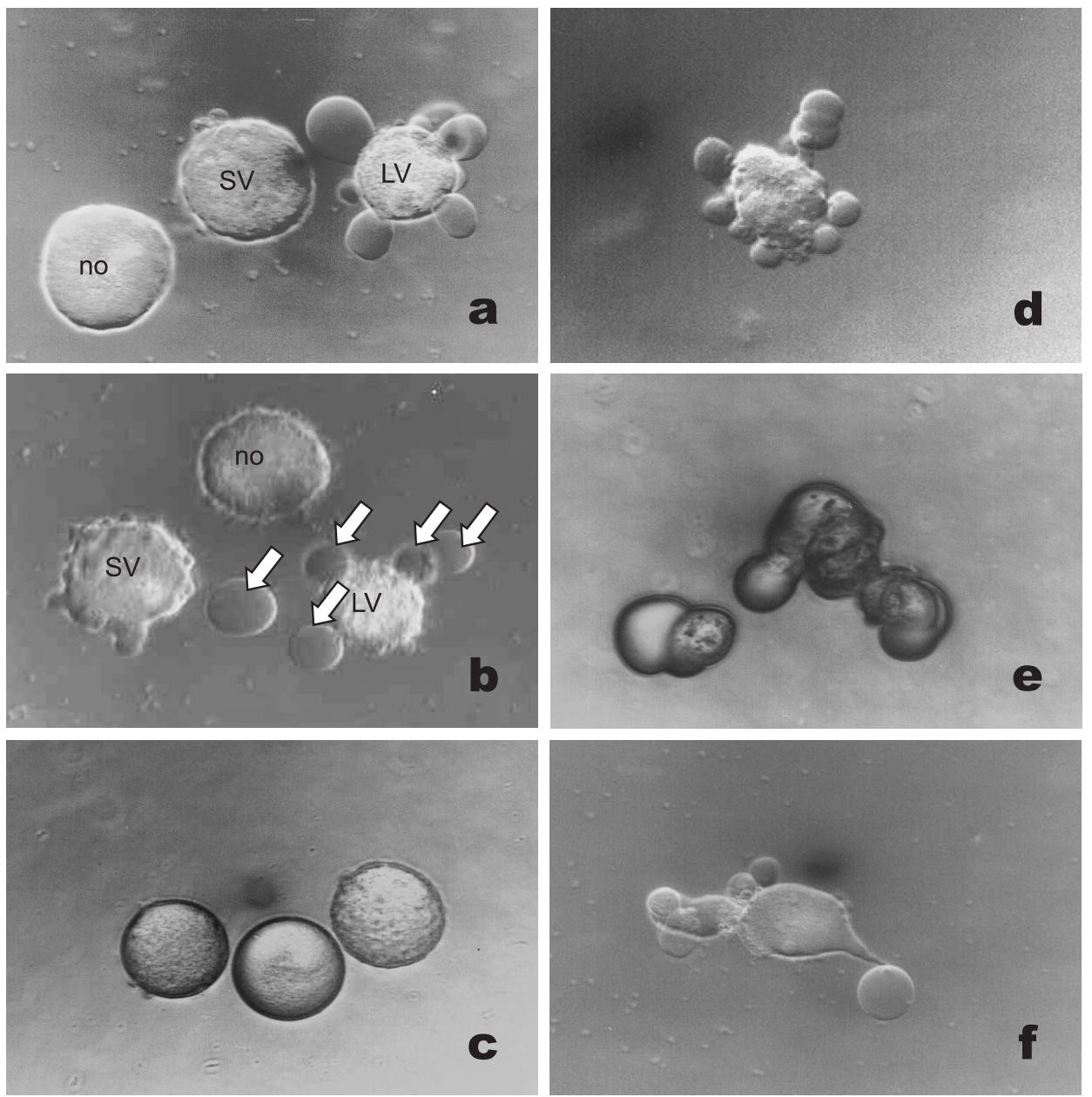

Figure 2. Effects of okadaic acid (OA) on cellular morphology of human blastomeres. (a) Different response of cells (3-cell fragmented D2 embryo) after $2 \mathrm{~h}$ in $6 \mathrm{mM}$ OA: LV - the blastomere with large vesicles of the plasma membrane (PM), probably lacking any organelles; SV - the blastomere with smaller PM exvaginations; no - the blastomere showing no overt sights of alteration. (b) The same cells $20 \mathrm{~min}$ after replacement into a drug free medium - the process of alteration continued, and the large PM vesicles frequently separated from the cells (arrows). (c) The same cells after $2 \mathrm{~h}$ in OA free culture - the PM changes disappeared or vesicles were freed from the cells, and NEBD took place. (d), (e), (f) The blastomeres from 6-cell uneven D4 embryo after the same procedure, which did not recover in a culture medium - substantial alteration of PM with polarization of cytoplasmic organelles, leaving large parts of cells filled only with the cytosol, caused lysis of the cells within $1 \mathrm{~h}$ after removal from the OA medium. 
found after fixation within $4 \mathrm{~h}$. Okadaic acid strongly affected the behavior of the cytoskeleton and plasma membrane properties of treated cells (Fig. 2).

All chromosome spreads were G-banded. In the worst cases, only a very primitive banding pattern was obtained, i.e. in most chromosomes only two or three chromodomains were apparent (Figs. 3e and 3f), and very rarely some individual chromosome could be completely classified. Centromere locations were not recognized roughly in $60 \%$ chromosomes. On the other side, chromosomes were in majority suitable for counting. Further data on drug-induced condensed chromosome banding patterns and single blastomere karyotypes will be published elsewhere after collecting much more spreads.

12 of 24 obtained chromosome spreads were subjected to one round of FISH analysis. In all 12 cases we obtained clear spot signals $(4 \times \mathrm{XX}, 2 \times \mathrm{X} 0,3 \times \mathrm{XY}, 2 \times \mathrm{XYY}$ and $1 \times 0 Y)$. FISH analysis was the less problematic step either in poorly condensed chromosomes (Fig. 4).

\section{DISCUSSION}

\subsection{Blastomere biopsy}

The normal survival and further development of biopsied embryos is a prerequisite in achieving the pregnancy after PGD. On the other side, the survival of isolated blastomeres is essential for successful genetic testing. Several biopsy methods have been tested till now in human AR centers. They differ in their effectiveness in accordance with the used cell removal technique. Here we present the experimental method that offers some potential advantages over the clinically used methods.

We suppose the PZD-based displacement blastomere biopsy to be more desirable and safer than making a relatively large circular opening in ZP with Tyrode's solution or laser. After the cell expulsion, a spontaneous $\mathrm{ZP}$ reassembly prevents the direct communication between the intrazonal space and the surrounding medium during prolonged cultivation while genetic tests are made. Thus, the biological function of ZP is minimally perturbed after this new type of embryo biopsy.

Moreover, the dissected narrow slit enables the cell separation even in slightly compacting embryos; the $\mathrm{ZP}$ constriction itself is able to gently disconnect conglued blastomeres in a few minutes. A positive effect of PZD upon the hatching process has been described [6]. Furthermore, the displacement method requires only one biopsy pipette; no pipette change, no pipette beveling, and no pipette cutting to a precise diameter are needed, in opposite to aspiration approaches.

This biopsy method was developed as a simple, easy and cheap tool for research purposes. In this work, the further viability of spare embryos is not important, but preliminary viability tests were performed, of course. In brief, mouse biopsied embryos and their individual isolated blastomeres were returned to the culture and evaluated later for their development and integrity, respectively. Mouse model has shown that blastomeres isolated using this method were able to divide synchronously with biopsied embryos that normally reached the blastocyst stage (data not shown). This method has not yet been tested for a clinical use.

\subsection{Okadaic acid treatment-induced chromosome condensation}

MPF (maturation promoting factor) is known as the key cell cycle regulator in $\mathrm{G}_{2} / \mathrm{M}$ transition and $\mathrm{M}$ phase control (reviewed in Musunuru and Hinds [20]). It consists of $\mathrm{p} 34^{\mathrm{cdc} 2} \mathrm{Ser} / \mathrm{Thr}$ protein kinase (histone H1 kinase) complexed with a regulator phosphoprotein - cyclin B1. The complete set of MPF downstream substrates is 


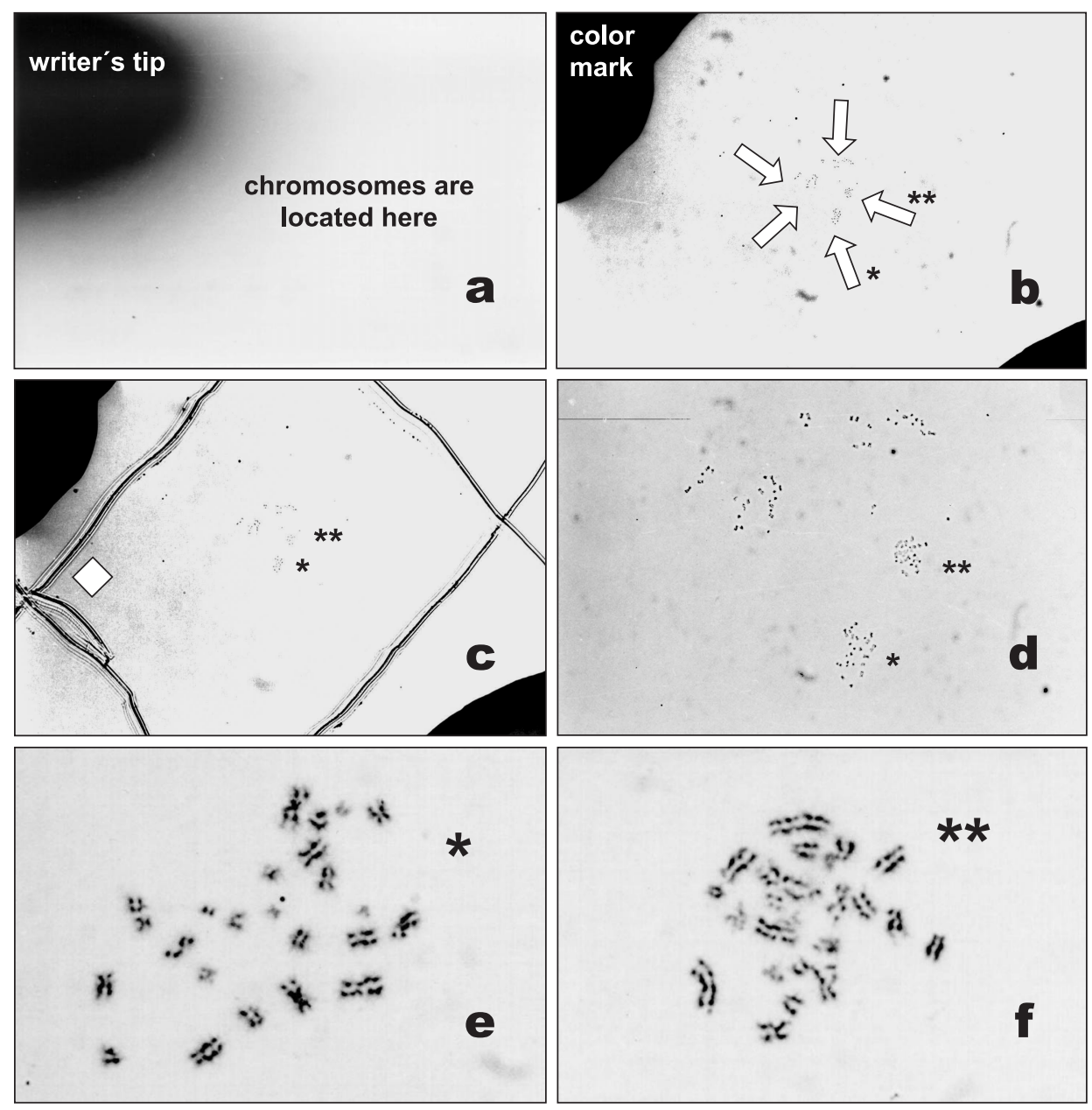

Figure 3. Manipulations with a single chromosome spread fixed on a microscopic slide I. Initial orientation and G-banding. Chromosome spreads (arrows) obtained from one multinucleated blastomere after the okadaic acid treatment; two of them are marked *, ** to find them easily on various pictures. (a) Chromosomes are found in a roughly pre-marked circle; their position is then highlighted manually with a fine waterproof writer. $/ 100 \times /$ (b) Chromosomes and color marks. /100×/ (c) Keeping in mind the mutual positions of color marks and the chromosomes, four perpendicular lines can be engraved manually, just looking with a free eye at the light reflex on the slide surface. The resulting miniature square area will help us to quickly find the spread under various circumstances. $/ 100 \times /$ (d) The same fixed chromosomes. $/ 400 \times /$ (e), (f) Detailed look at the banded spreads * and **. Prematurely condensed chromosomes are of a relatively poor morphology with a very primitive G-banding pattern, but they may be at least counted or further analyzed e.g. by the means of FISH (numeric aberrations as far as translocations can be diagnosed much more accurately than in interphase nuclei). /1 000×/ 

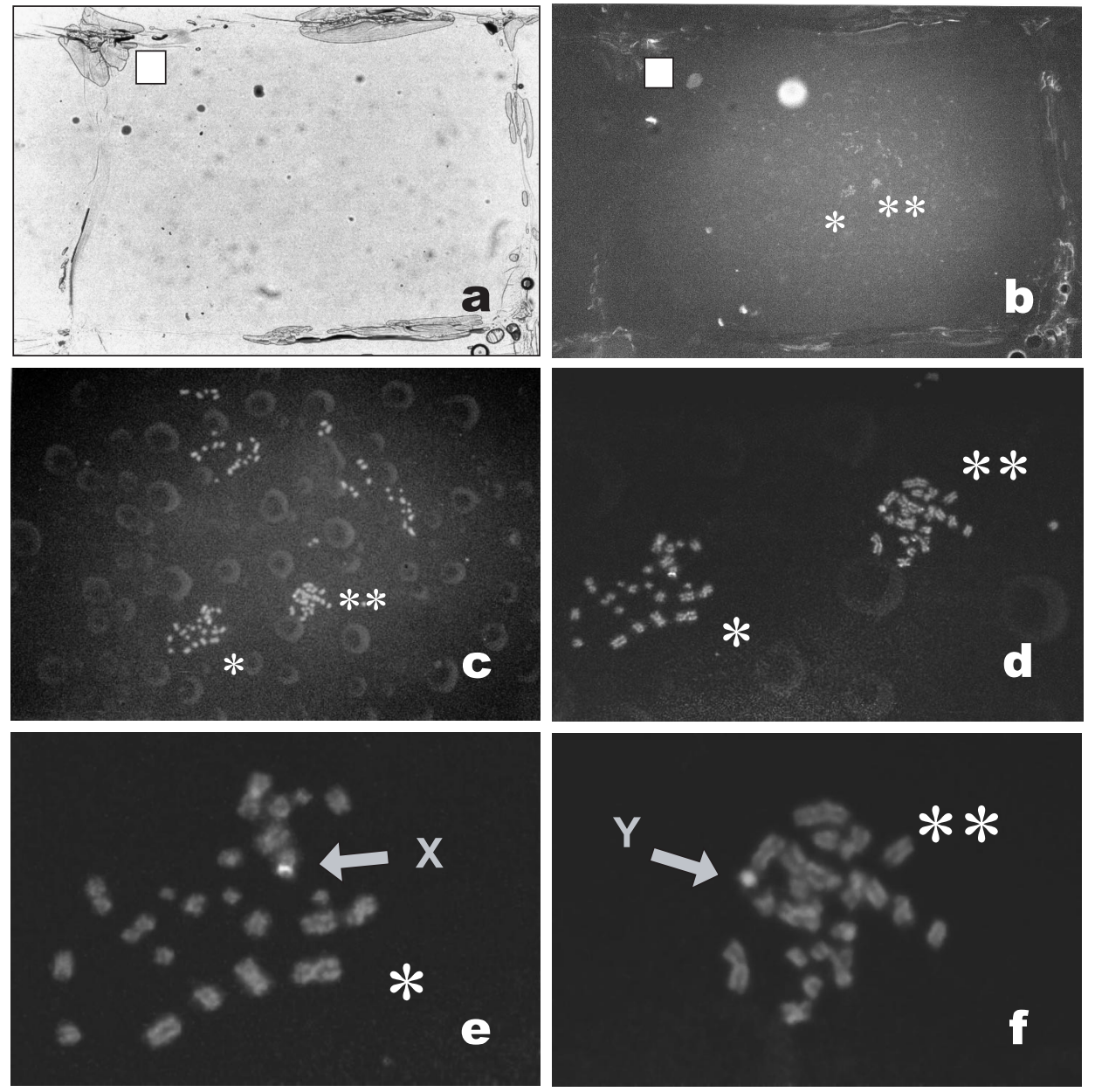

Figure 4. Manipulations with a single chromosome spread fixed on a microscopic slide II. After G-banding, the same chromosomes from Figure 3 were hybridized with fluorescent probes for sex chromosomes. White squares indicate the position and rotation of the upper left corner of the engraved helper quadrate; compare with Figure 3c. (a) Chromosomes mounted in the antifade solution are immersed and thus invisible either in phase contrast. But they were easily located accordingly to their position in the engraved square. /100 $\times /$ (b) We quickly found the chromosomes after UV illumination, simply refocusing the microscope. Without the precise knowledge of their position, the chromosomes could be overseen with a high probability. $/ 100 \times /$ (c), (d) The same view, magnified. (e), (f) Detailed look at the FISH results, the X chromosome centromere (red signal) and the Y chromosome q arm distal heterochromatin (green signal) were unambiguously detected despite the poor morphology of okadaic acid-induced prematurely condensed chromosomes. /1 000×/ 
still unknown, but MPF is clearly responsible for initiation of early mitotic events (nuclear envelope breakdown (NEBD), nuclear lamina disassembly, chromosome condensation and spindle formation). The quick drop down of MPF activity is vital for the metaphase to anaphase transition.

Okadaic acid is a potent Ser/Thr protein phosphatase inhibitor $\left(\mathrm{IC}_{50}\right.$ for type $2 \mathrm{~A}$ is about $0.1 \mathrm{nM}$, for type 1 about $12 \mathrm{nM}$, for type $2 \mathrm{~B}$ about $5 \mu \mathrm{M}$; type $\mathrm{C}$, alkaline, acid and tyrosine phosphatases are not affected at concentrations about $10 \mu \mathrm{M}$ ). OA was referred to induce the maturation of oocytes maintained arrested in dictyotene. After OA injection, immature oocytes rapidly showed NEBD, lamin depolymerization, and chromosome condensation, accompanied with a rapid appearance of MPF activity, and with a burst of protein phosphorylation in Xenopus and mouse [21, 22]. These experiments also showed a full biological effectivity (tested by injection into other oocytes) of MPF appeared after the OA treatment either in oocytes with completely blocked proteosynthesis, indicating that preformed inactive MPF existed in their ooplasms.

We can not exactly explain our findings that D3 and D4 blastomeres responded less desirable to OA treatment than D1-D2 cells. To our knowledge, no comparable data touching drug-induced chromosome condensation in D2 and older blastomeres exist in literature. Besides lymphocytes and solid tumors (e.g. [5, 8]), all studies were focused to oocytes, polar bodies and/or D1 embryos (zygotes) (e.g. [1, 4, 9, 11, 12, 15, 21-23, 25]). We speculate that ooplasmic store of plenty of kinds of molecules and organelles may play a certain role. During the first days of development, the ooplasm is divided into cytoplasms of individual blastomeres in cleaving embryos, and many molecules are consumed by intensive replicative processes. On D3, the critical transition to significant transcription of the embryonic genome takes place, and attenuated human embryos may be more sensitive to OA toxic effects in this period.
Furthermore, the chromosome condensation is an energy consuming process dependent on ATP and on a lot of nuclear proteins. Inappropriately timed increase in energy or protein requirements may result in an abortive metabolic situation in the cytoplasm. Also, aberrant embryos gradually loss their developmental potential, and nonviable cells are accumulated, resulting in a cleavage arrest and/or embryo death. Arrested or dying blastomeres are probably not able to satisfy the chromosome condensation requirements. Mitochondrial function and apoptosis were not monitored in our experiments.

Chromosomes obtained from blastomeres after OA treatment were of a variable quality. Besides the factors dependent on the embryo quality mentioned above, also cell cycle-associated problems may play a substantial role in chromosome condensation. Blastomeres of early human embryos loss their exact mutual mitotic synchrony, it is not possible to estimate the cell cycle stage of any individual biopsied cell since D3. After biopsy, some blastomeres may be fixed in the time of their natural $M$ phase, some others can be forced to $\mathrm{M}$ phase with OA, because the optimal level of pre-MPF is present in the cytoplasm. The absence or a low level of effector proteins in cells at remaining cell stages $\left(G_{1}, G_{0}, S\right.$, early $\left.G_{2}\right)$ may be the cause of a poor quality of the majority of chromosome spreads obtained from individual blastomeres. Chromatin fragmentation and pulverization usually occurs in S phase cells treated with phosphatase inhibitors [5], probably due to presence of replication-associated DNA gaps. From the studies on lymphocytes there is known that $G_{0}$ cells do not undergo the drug-induced chromosome condensation [8]. Long lasting blastomere diagnostic procedures are not desirable for PGD, but a minimal artificial cell cycle synchronizing influence seems to be inevitable for a further research.

Our experiments were inspired by very promising results in the field of experimental 
oncology and biodosimetry $[5,8]$, with the use of tumor and somatic cell lines. Some cell types gave more than $90 \%$ nuclei with condensed chromosomes in a short time after the treatment with phosphatase inhibitors. But on the other side, some lines showed a very poor or no response. Finally, some cell lines were completely lysed. Our results indicate that D1 to D2 embryonic cells are middle category responders, while older blastomeres are poor responders in the terms of surviving and successfully condensed chromosomes. A critical evaluation of all technical steps given here, and a comparison of our results with results obtained in different laboratories is needed prior making any definitive conclusions touching this question. The new study carried on unaffected embryos donated for research may help to answer the question, whether spare embryos are more susceptible to the cell death in the OA medium, or whether this is the case of a general feature characteristic for a given cell type - similarly like in biodosimetry studies.

From the theoretical aspect, the unique role of MPF in chromosome condensation is questionable. Firstly, it is known that mitotic cyclins, including MPF subunit cyclin B, appear mainly in the late $G_{2}$ phase. Secondly, there was drug-induced chromosome condensation observed in cells lacking both p34 ${ }^{\text {cdc2 }}$-cyclin B kinase activity and histone H1 hyperphosphorylation [5, 13]. More experimental data from the basal molecular research are needed to elucidate this field of interest.

We conclude that a substantial improvement in this area of research is needed prior to a clinical application of the individual blastomere karyotypization. The crucial point is the induction of an appropriate chromosome condensation. From this point of view, unperturbed viability of biopsied cells during induction procedures is the essential condition. Although cytochalasin B prevented in part the negative effect of OA upon cytoskeletal changes (data not shown), the occurrence of these abnormalities is still detrimental for many of biopsied blastomeres, and represents a major limiting factor for successful chromatin condensation. Prematurely condensed chromosomesbased karyotypization itself also requires further improvement and more detailed study. FISH signal detection was successful in $100 \%$ cases of premature spreads and it does not seem to be a problem in this context. Currently, the presented method may be used for increasing the number of metaphase-like cells in experimental studies on genetic constitution of spare embryos.

We have shown that chemically induced prematurely condensed chromosomes are suitable for repeated use in subsequent diagnostic procedures, G-banding and FISH analysis, without apparent chromatin loss. After avoiding the drastic cell loss due to toxic side effects of $\mathrm{OA}$, the drug-induced chromosome condensation step could be used prior to FISH analysis to increase the probability of obtaining enumerable chromosome spreads. Similarly like in experimental oncology and biodosimetry, countable chromosomes of a poor morphology are always a better result than having only the interphase nuclei. In unsuccessful cases with no or incomplete drug-induced chromosome condensation, fixed chromatin can be evaluated by FISH, similarly like interphase nuclei are analyzed during conventional PGD.

\section{ACKNOWLEDGEMENTS}

This work is a part of the grant project IG 1/1998 "Predimplantačná genetická diagnostika" funded by the Medical Faculty of P.J. Šafárik University, Košice, Slovakia. This work was also supported by the grant KLV 45/97 from the Health Care Ministry of the Slovak Republic. Many thanks to the clinical cytogenetic laboratory leaded by Dr. Nebesňáková, University Hospital, Tr. SNP 1, Košice, and to Dr. I. Černáková from the Slovak Postgraduate Academy of Medicine, Bratislava, for a help with chromosome banding. Thank to Dr. E. Kočiš ová and A. Plichtová for a technical help. 


\section{REFERENCES}

[1] Alexandre H., Van Cauwenberge A., Tsukitani Y., Mulnard J., Pleiotropic effect of okadaic acid on maturing mouse oocytes, Development 112 (1991) 971-980.

[2] Balicek P., Forejt J., Rubes J., Metody analyzy chromozomu, Cytogeneticka sekce Ceskoslovenske biologicke spolecnosti pri CSAV, Brno, 1988.

[3] Cassel M.J., Munne S., Fung J., Weier H.-U.G., Carrier specific breakpoint spanning DNA probes: an approach to preimplantation genetic diagnosis in interphase cells, Hum. Reprod. 12 (1997) 2019-2027.

[4] Cieslak J., Evsikov S., Ivakhnenko V., Strom C., Kuliev A., Verlinsky Y., Preimplantation diagnosis for maternally and paternally derived translocations using conversion of single blastomeres and second polar bodies into metaphase chromosomes, Abstracts of the 16th Annual meeting of the ESHRE, Bologna, Italy 2000 , Hum. Reprod. 15 (2000) Abstract Book 1, 48-49.

[5] Coco-Martin J.M., Begg A.C., Detection of radiation-induced chromosome aberrations using fluorescence in situ hybridization in drug induced premature chromosome condensation of tumour cell lines with different radiosensitivities, Int. J. Radiat. Biol. 71 (1997) 265-273.

[6] Cohen J., Feldberg D., Effects of the size and the number of zona pellucida openings on hatching and trophoblast outgrowth in the mouse embryo, Mol. Reprod. Dev. 30 (1991) 70-78.

[7] Darroudi F., Fomina J., Meijers M., Natarajan A.T., Kinetics of the formation of chromosome aberrations in X-irradiated human lymphocytes, using PCC and FISH, Mutation Research 404 (1998) 55-65.

[8] Durante M., Furusawa Y., Gotoh E., A simple method for simultaneous interphase-metaphase chromosome analysis in biodosimetry, Int. J Radiat. Biol. 74 (1998) 457-462.

[9] Dyban A.P., De Sutter P., Verlinsky Y., Okadaic acid induces premature chromosome condensation reflecting the cell cycle progression in one-cell stage mouse embryos, Mol. Reprod. Dev. 34 (1993) 402-415.

[10] Findlay I., Matthews P., Quirke P., Multiple genetic diagnoses from single cells using multiplex PCR: reliability and allele dropout, Prenat. Diagn. 18 (1998) 1413-1421.

[11] Gavin A.C., Tsukitani Y., Schorderet-Slatkine S., Induction of M-phase entry of prophaseblocked mouse oocytes through microinjection of okadaic acid, a specific phosphatase inhibitor, Exp. Cell. Res. 192 (1991) 75-81.
[12] Gavin A.C., Vassalli J.D., Cavadore J.C., Schorderet-Slatkine S., Okadaic acid and p13 $3^{\text {suc } 1}$ modulate the reiniation of meiosis in mouse oocytes, Mol. Reprod. Dev. 33 (1992) 287-296.

[13] Guo X.W., Th'ng J.P., Swank R.A., Anderson H.J., Tudan C., Bradbury E.M., Roberge M., Chromosome condensation induced by fostriecin does not require $\mathrm{p} 34^{\mathrm{cdc} 2}$ kinase activity and histone $\mathrm{H} 1$ hyperphosphorylation, but is associated with enhanced histone $\mathrm{H} 2$ and $\mathrm{H} 3$ phosphorylation, EMBO J. 14 (1995) 976-985.

[14] Hlinka D., Herman M., Vesela J., Hredzak R. Horvath S., Pacin J., A modified method of intracytoplasmic sperm injection without the use of polyvinylpyrrolidone, Hum. Reprod. 13 (1998) 1922-1927.

[15] Jacquet P., de Saint-Georges L., Barrio S. Baugnet-Mahieu L., Morphological effects of caffeine, okadaic acid and genistein in one-cell mouse embryos blocked in $\mathrm{G}_{2}$ by $\mathrm{X}$-irradiation, Int. J. Radiat. Biol. 67 (1995) 347-358.

[16] Johnson R.T., Rao P.N., Mammalian cell fusion Induction of premature chromosome condensation in interphase nuclei, Nature 226 (1970) 717-722.

[17] Liu J., Tsai Y.L., Zheng X.Z., Yazigi R.A., Baramki T.A., Compton G., Katz E., Feasibility study of repeated fluorescent in situ hybridization in the same human blastomeres for preimplantation genetic diagnosis, Mol. Hum. Reprod. 4 (1998) 972-977.

[18] MacLean-Fletcher S., Pollard T.D., Mechanism of action of cytochalasin B on actin, Cell 20 (1980) 329-341.

[19] Muggleton-Harris A.L., Glazier A.M., Pickering S., Wall M., Genetic diagnosis using polymerase chain reaction and fluorescent in situ hybridization analysis of biopsied cells from both the cleavage and blastocyst stages of individual cultured human preimplantation embryos, Hum. Reprod. 10 (1995) 183-192.

[20] Musunuru K., Hinds P.W., Cell cycle regulators in cancer, S. Karger AG, Basel, 1997.

[21] Rime H., Ozon R., Protein phosphatases are involved in the in vivo activation of histone $\mathrm{H} 1$ kinase in mouse oocyte, Dev. Biol. 141 (1990) 115-122.

[22] Rime H., Huchon D., Jessus C., Goris J., Merlevede W., Ozon R., Characterization of MPF activation by okadaic acid in Xenopus oocyte, Cell. Differ. Develop. 29 (1990) 47-58

[23] Schwartz D.A., Schultz R.M., Stimulatory effect of okadaic acid, an inhibitor of protein phosphatases, on nuclear envelope breakdown and protein phosphorylation in mouse oocytes and one-cell embryos, Dev. Biol. 145 (1991) 119-127. 
[24] Tarkowski A.K., An air drying method for chromosome preparations from mouse eggs, Cytogenetics 5 (1966) 394-400.

[25] Verlinsky Y., Kuliev M., Cieslak J., De Sutter P., Dyban A., Ginsberg N., Lifchez A., Rechitsky S. Strom C.M., Preimplantation diagnosis of genetic diseases, Wiley-Liss Inc., New York, 1993.
[26] Wimmers M.S.E., van der Merve J.V., Chromosome studies on early human embryos fertilized in vitro, Hum. Reprod. 3 (1988) 894-900.

[27] Zenzes M.T., Casper R., Cytogenetics of human oocytes, zygotes and embryos after in vitro fertilization, Hum. Genet. 88 (1992) 367-375. 